\title{
Heritability and Familiality of Temperament and Character Dimensions in Korean Families with Schizophrenic Linkage Disequilibrium
}

\author{
Byung Dae Lee $e^{1,2,3}$, Je Min Park ${ }^{1,2,3}$, Young Min Lee ${ }^{1,2,3}$, Eunsoo Moon ${ }^{1,2,3}$, Hee Jeong Jeong ${ }^{1,3}$, Young In Chung ${ }^{2}$, \\ Young Mi Yi ${ }^{1,3}$ \\ 'Department of Psychiatry, Pusan National University Hospital, ${ }^{2}$ Department of Psychiatry, Pusan National University College of Medicine, \\ ${ }^{3}$ Medical Research Institute, Pusan National University Hospital, Busan, Korea
}

\begin{abstract}
Objective: Categorical syndromes such as schizophrenia may represent complexes of many continuous psychological structural phenotypes along several dimensions of personality development/degeneration. The present study investigated the heritability and familiality of personality dimensions in Korean families with schizophrenic linkage disequilibrium (LD).

Methods: We recruited 179 probands (with schizophrenia) as well as, whenever possible, their parents and siblings. We used the Temperament and Character Inventory (TCl) to measure personality and symptomatic dimensions. The heritability of personality dimensions in a total of 472 family members was estimated using Sequential Oligogenic Linkage Analysis Routines (SOLAR). To measure familiality, we compared the personality dimensions of family members with those of 336 healthy unrelated controls using analysis of variance (ANOVA) analysis.

Results: Three of the seven TCl variables were significantly heritable and were included in subsequent analyses. The three groups (control, unaffected first-degree relative, case) were found to significantly differ from one another, with the expected order of average group scores, for all heritable dimensions.

Conclusion: Despite several study limitations with respect to family recruitment and phenotyping, our results show that aberrations in several personality dimensions related to genetic-environment coactions or interactions may underlie the complexity of the schizophrenic syndrome.
\end{abstract}

KEY WORDS: Heritability; Familiality; Personality; Dimension; Schizophrenia.

\section{INTRODUCTION}

Schizophrenia is the most devastating mental illness and can lead to deterioration in the social and occupational functioning of affected individuals, ${ }^{1,2)}$ resulting in a significant cost to society. ${ }^{3,4)}$ A wide range of studies suggest a genetic component to the etiology of this disorder. ${ }^{5)}$ Although family, twin, and adoption studies have provided strong evidence that genetic variation plays a major role in the etiology of schizophrenia, susceptibility genes have proven difficult to identify in a definitive manner.

A substantial number of researchers in the domain of neuroscience are devoted to elucidating schizophrenia's causes, particularly with respect to basic genetics. How-

\footnotetext{
Received: April 10, 2015 / Revised: July 3, 2015

Accepted: July 14, 2015

Address for correspondence: Je Min Park, MD, PhD

Department of Psychiatry, Pusan National University Hospital, 179

Gudeok-ro, Seo-gu, Busan 49241, Korea

Tel: +82-51-240-7306, Fax: +82-51-248-3648

E-mail: psyleebd@gmail.com
}

ever, the mystery posed by this disorder is consistent with the mystery posed by the brain in general, and advances in understanding schizophrenia and its causes have been meager relative to the amount of research effort that has been devoted to the problem. A recent study conducted with a large and heterogeneous sample of subjects of European ancestry proved discouraging, suggesting that 14 genes that were previously believed, based on replicable results, to contribute to the susceptibility to schizophrenia, may in fact play little causal role in the disease. ${ }^{6}$

However, much work remains at both the population and molecular levels before genes and their connection to schizophrenia can be dismissed. Many researchers have suggested that alternative methods of phenotyping should be explored. It is very clear that there are limitations to categorical phenotypes, such as those in the Diagnostic and Statistical Manual of Mental Disorders, 4th edition (DSM-IV) system. Numerous researchers are exploring comparable quantitative endophenotypes as alternatives to classical qualitative phenotypes to represent schizophrenia.

(a) This is an Open-Access article distributed under the terms of the Creative Commons Attribution Non-Commercial License (http://creativecommons.org/licenses/by-nc/4.0) which permits unrestricted non-commercial use, distribution, and reproduction in any medium, provided the original work is properly cited. 
Mode-of-inheritance studies have suggested that multiple genes are likely involved in the etiology of schizophrenia. Given that the effect of any single gene is generally modest for this disorder, we speculate that it may be more productive to identify the clinical features that tag subtypes that are quite genetically homogeneous, thereby facilitating gene identification. Clinical subtyping has been an effective method for determining the etiology of other illnesses, such as Alzheimer's disease and breast cancer; in these cases, families with early onset illness led researchers to the identification of disease genes. ${ }^{7,8)}$

Several clinical features have been shown to augment evidence of a genetic linkage to chromosomal regions or an association with gene variants. Comorbid panic disorder $^{9)}$ and bipolar II disorder ${ }^{10)}$ appear to enhance linkage to distinct regions on chromosome 18q. In two datasets, psychotic features showed linkage to chromosome $13 \mathrm{q}^{11,12)}$ and early age at onset showed linkage to chromosome $21 \mathrm{q} 22$ in two cohorts. It has been reported that mania at onset enhances linkage to chromosome 16p, and a linkage to chromosome 2 was shown to be associated with attempted suicide in bipolar disorder. ${ }^{13)}$ Psychotic features, mood-incongruent psychotic features, and persecutory delusions in bipolar disorder have strengthened evidence for a genetic association with dysbindin (DTNBP1), neuregulin (NRG1), and D-amino acid oxidase activator (DAOA, G72). ${ }^{14)}$ These early successes suggest that clinical phenomenology can help to define more genetically homogenous forms of schizophrenia.

The choice of features studied in schizophrenia genetics has been guided largely by clinical experience. Features that show familial aggregation may be particularly promising ${ }^{15)}$ and, as mentioned previously, most of the features that enhance linkage or association signals are indeed familial. However, only a minority of the myriad clinical features of schizophrenia have been studied.

The study of clinical features has been limited by the time-consuming process of gathering and assembling relevant clinical data in cohorts of sufficient size. Largescale genetics efforts have yielded the human genome sequence and, more recently, the HapMap, which catalogues the common patterns found in human sequence variation. The authors of the HapMap paper called for comparable large-scale efforts in the phenotypic arena. A similar concept, the "Human Phenome Project," was advanced by Freimer and Sabatti, ${ }^{16)}$ who advocated for the development of an international effort to create phenomic databases in the form of comprehensive assemblages of systematically collected phenotypic information to aid in the identification of disease genes. In this regard, the Autism Phenome Project has begun the prospective compilation of comprehensive phenotypic data with the aim of parsing genetic heterogeneity in autism, ${ }^{17)}$ and the Epilepsy Phenome-Genome Project was initiated to accomplish similar goals (http://65.175.48.5/epgp/index.htm). The aforementioned study to establish a Bipolar Disorder Phenome Database could be extended to establish a similar Korean Schizophrenia Phenome Database. To our knowledge, no such databases currently exist for schizophrenia.

One possible causal mechanism for schizophrenia may be the presence of genetic aberrations in psychological dimensions, which can be viewed as sub(endo)phenotypes. Categorical syndromes, such as schizophrenia, may be complexes of many continuous phenotypes for mental structures that exist along several dimensions of personality development/degeneration. Quantitative endophenotypes are needed to better understand the pathogenesis of schizophrenia. This concept was reflected in the recent publication of the Diagnostic and Statistical Manual of Mental Disorders, 5th edition (DSM-5), which included the category schizophrenia spectrum and other psychotic disorders.

Traditionally, personality has been regarded as the basis for psychiatric symptoms, as reflected in previous DSM versions. This notion is currently changing, with this shift indicated in the new DSM-5, which no longer includes axis II diagnoses as the bases of axis I psychiatric symptoms. Psychiatric diagnosis faces numerous challenges in the current era, in which the brain's complexity is acknowledged but not well understood. The existing categorical phenotypic boundaries have become more ambiguous. Personality is currently regarded as the mental structure giving rise to psychiatric symptomatology; in fact, the various personality disorders are closely related to specific psychiatric diagnoses. Hence, in the current paradigm shift involving psychiatric diagnoses, personality dimensions may be continuous with psychological dimensions. To clarify this relationship, we aimed to explore the heritability of temperament and character dimensions in schizophrenic families. ${ }^{18-21)}$

Of particular relevance is the fact that many models suggest that personality dimensions are heritable and influence the genetic loading of schizophrenia. Personality traits are under genetic influence, and the heritability of personality reaches levels of $30 \%$ to $60 \%$. Personality exhibits a high familial tendency in the general population and in various psychiatric disorders, including major depression, alcohol dependence, and bulimia. ${ }^{22-26)}$ In addi- 
tion to neuropsychological or neurobiological characteristics, the biogenetic components of personality may also be considered endophenotypes of schizophrenia if they can be found both in schizophrenic probands and their first-degree relatives. ${ }^{27)}$

The psychobiological model of personality suggests that an individual's temperament is heritable and regulated by neurotransmission linked to the pathophysiology of schizophrenia. ${ }^{28,29)}$ More recently, there has been evidence to suggest that dimensions of character are also heritable and may influence the risk for schizotypy. ${ }^{30)}$ Despite conflicting results, the temperament of harm avoidance has been identified as subject to genetic loading. The present study aimed to investigate the heritability and familiality of dimensions of temperament and character in Korean families with psychotic members.

\section{METHODS}

We recruited 179 probands (with schizophrenia) as well as, whenever possible, their parents and siblings. To identify the best possible diagnosis, we used medical records and Korean versions of the Diagnostic Interview for Genetic Studies (DIGS) and the Family Interview for Genetic Studies (FIGS). We also used the Temperament and Character Inventory (TCI) to measure various dimensions of temperament and character. The TCI is an inventory for evaluating distinct personality traits. It is closely related to the Tridimensional Personality Questionnaire (TPQ) and also derives elements from Zuckerman's and Eysenck's Dimensions of Personality. The TCI addresses seven dimensions of personality traits, with four so-called "temperaments" (novelty seeking, harm avoidance, reward dependence, and persistence) and three "characters" (selfdirectedness, cooperativeness, and self-transcendence). ${ }^{31)}$

\section{Sample Selection}

All subjects were recruited in accordance with the principles of the Declaration of Helsinki and with approval from the Institutional Review Boards of Pusan National University Hospital. Probands were recruited independently from Pusan National University Hospital (Busan, Korea). All probands had a disease onset of no later than age 40 years, a history of at least one psychiatric hospitalization, a discharge diagnosis of schizophrenia, and a Korean surname. Parents and first-degree relatives of probands were also recruited wherever possible to allow for the determination of genetic phase and for family-based linkage disequilibrium analyses; if additional relatives with psychotic disorders were identified, efforts were made to recruit these relatives as well. Each subject was interviewed by a trained bilingual psychiatrist, who was blind to the history of the subject, using the Korean version of the DIGS. ${ }^{32)}$ Medical records (inpatient and outpatient) were summarized. An interview with a close relative, using a Korean version of the FIGS was also completed for each subject. ${ }^{33)}$ We analyzed data from 179 probands and their available family members. All affected subjects (i.e., those given formal psychiatric diagnoses in the present study) within each family were diagnosed using a best-estimate diagnostic process, as described by Walss-Bass et $a l^{34)}$ The process yields a lifetime consensus diagnosis or diagnoses using the DSM-IV. Data from 472 subjects from 179 families were analyzed in this paper; 179 probands had Axis I disorders, as determined by consensus DSM-IV diagnoses for schizophrenia.

A total of 179 subjects had a history of psychosis, operationalized in the present study as the presence of at least one of the following symptoms at some point in the subject's lifetime: hallucinations, delusions, grossly disorganized thought processes, or grossly disorganized behavior. This definition of psychosis corresponds to four of the five symptoms and signs listed under Criteria A of the DSM-IV definition of schizophrenia. We also considered a history of "negative symptoms" (abulia, alogia, avolition), also listed under Criteria A of the DSM-IV definition of schizophrenia, as sufficient for a diagnosis of psychosis. For each subject, the best estimators also diagnosed whether manic syndromes or episodes had been present during the course of the disorder. The distinction between schizophrenia and schizoaffective disorder followed the criteria of the DSM-IV, as operationalized by Walss-Bass et al. ${ }^{34)}$

Our study included 336 Korean control subjects who were psychiatrically normal and belonged to the age groups specified in the sample. Subjects with potentially biasing conditions were excluded from the study. Subjects were also excluded if they had been receiving corticoid, estrogen, androgen, or T3 (triiodothyronine)-T4 (thyroxine) therapy or diphenylhydantoin, vitamin $\mathrm{D}$, bisphosphonate, calcitonin, fluoride, thiazide diuretics, or barbiturates for more than 6 months, as all these drugs may yield biasing effects. Because it was impossible to obtain a population-based register for technical and legal reasons, control subjects meeting the inclusion/exclusion criteria were identified from among volunteers (students and hospital workers) at the hospital. 


\section{Statistical Analysis}

We estimated the heritability of the various temperament and character dimensions measured in the 472 family members using Sequential Oligogenic Linkage Analysis Routines (SOLAR). To determine familiality, we compared the temperament and character dimensions of family members with those of 336 healthy unrelated controls using analysis of variance (ANOVA) (SPSS software, version 11.5; SPSS Inc., Chicago, IL, USA). We also used SOLAR to investigate correlations between genetic/environmental variables and the symptoms associated with significant temperament and character dimensions, with the significance level set at $5 \%$.

\section{RESULTS}

\section{Heritability}

Three of the seven TCI variables (harm avoidance, cooperativeness, self-transcendence) were significantly heritable and were included in subsequent analyses. Heritability was the highest for self-transcendence $(0.34, p=0.0006)$, intermediate for harm avoidance $(0.28, p=0.006)$, and the lowest for cooperativeness $(0.24, p=0.01)$. The remaining four TCI variables (novelty seeking, reward dependence, persistence, self-directedness) were not significantly heritable and were therefore excluded from subsequent analyses. Heritability was nominally high for reward dependence (0.15) and self-directedness (0.13) (Table 1).

\section{Familial Aggregation}

The control group, the unaffected group of first-degree relatives, and the case groups differed significantly, with the expected order of average group scores, for most heritable dimensions. High harm avoidance statistically differentiated the case group from the first-degree-relative and control groups $(p=0.000)$. Low self-transcendence differentiated the control group from the case and the first-degree relative groups $(p=0.000)$. Low cooperativeness differentiated the case group from the first-degree-relative group and the first-degree-relative group from the control group $(p=0.000)$ (Table 2).

\section{Genetic/Environmental Correlations}

Genetic/environmental correlations with the schizophrenic phenotype in terms of temperament and character dimensions are displayed in Table 3 . There were no genetic correlations with the schizophrenic phenotype for any temperament and character dimensions. Environmental correlations were suggested for harm avoidance $(p=0.0000272)$ and cooperativeness $(p=0.0001577)$.

Table 1. Heritability $(\mathrm{H} 2 \mathrm{r}$ ) of temperament and character measures

\begin{tabular}{lll}
\hline Personality dimension & H2r (SE) & p value \\
\hline Novelty seeking & $0.07(0.11)$ & 0.25 \\
Harm avoidance & $0.28(0.12)$ & 0.006 \\
Reward dependence & $0.15(0.12)$ & 0.10 \\
Persistence & $0.08(0.12)$ & 0.25 \\
Self directiveness & $0.13(0.14)$ & 0.17 \\
Cooperativeness & $0.24(0.11)$ & 0.01 \\
Self transcendence & $0.34(0.11)$ & 0.0006 \\
\hline
\end{tabular}

SE, standard error

Table 2. Familial aggregation of temperament and character measures

\begin{tabular}{|c|c|c|c|c|}
\hline Personality dimension & Control & Unaffected lst degree relative & Case & $p$ value \\
\hline Harm avoidance & $18.1 \pm 6.0$ & $18.4 \pm 5.8$ & $22.5 \pm 6.3^{*}+\dagger$ & 0.000 \\
\hline Cooperativenes & $31.6 \pm 5.8$ & $29.5 \pm 5.6^{*}$ & $26.7 \pm 6.9^{* \cdot \dagger}$ & 0.000 \\
\hline Self transcendence & $10.3 \pm 5.2$ & $12.1 \pm 5.9^{*}$ & $12.6 \pm 6.7^{*}$ & 0.000 \\
\hline
\end{tabular}

Values are presented as mean \pm standard deviation.

${ }^{*}$ Compared to controls by Scheffe test; ${ }^{\dagger}$ compared to 1 st degree relatives by Scheffe test.

Table 3. Bivariate analysis between affection status and temperament and character measures

\begin{tabular}{|c|c|c|c|c|c|}
\hline \multirow{3}{*}{$\begin{array}{l}\text { Personality } \\
\text { dimension }\end{array}$} & \multicolumn{5}{|c|}{ Schizophrenic phenotype } \\
\hline & \multicolumn{3}{|c|}{ Genetic correlation } & \multicolumn{2}{|c|}{ Environmental correlation } \\
\hline & $\rho g(\mathrm{SE})$ & $p(0)$ & $p(1$ or -1$)$ & $\rho \in(\mathrm{SE})$ & $p$ \\
\hline Harm avoidance & $0.9233085(\mathrm{NC})$ & 0.9374519 & 1 & $0.3871830(0.9984003)$ & 0.0000272 \\
\hline Cooperativeness & $-1(\mathrm{NC})$ & 0.5908300 & NC & $-0.3460180(0.1108247)$ & 0.0001577 \\
\hline Self transcendence & $-0.1366977(\mathrm{NC})$ & 1 & 0.5 & $0.1420517(0.0806877)$ & 0.0568722 \\
\hline
\end{tabular}

$\rho \mathrm{g}$, genetic correlation coefficient; SE, standard error; $\rho$ e, environmental correlation coefficient; NC, not computable. 


\section{DISCUSSION}

The schizophrenia phenome database is designed to complement the large body of available genetic data. The objective of the database is to accelerate the discovery of genes that contribute to schizophrenia, a common and often disabling disease. Providing public access is key to making the data valuable to the community. Value could be added to the schizophrenia phenome database by incorporating data from additional studies. It would also be useful to combine these clinical data with physiological evidence from studies involving brain imaging, evoked potentials, and neuropsychological data as well as with data from studies focusing specifically on the role of the hypothalamic-pituitary-adrenal axis. Further steps could be added to the existing dataset, including data reduction through techniques such as factor analysis. These factors could then be tested for degree of familiality, and the factor scores could be used as phenotypes for genetic study.

It is important but very difficult to recruit sufficient numbers of control subjects who are carefully matched for age and sex. One limitation of the present study is that the recruited normal controls may be excessively normal samples, despite the fact that we recruited from general populations. We could not implement for recruiting age/sex matched controls in the current study due to the numerous limitations involved in recruitment. We are currently designing a recruiting method to achieve matched controls. However, some researchers have expressed the opinion that it is legitimate to conduct genetic studies without age/sex adjustment in spite of possible population stratification by covariates or confounders.

Our results suggest that aberrations in several dimensions pertaining to temperament and character that are attributable to genetic-environment coactions or interactions may underlie the complexity of the schizophrenic syndrome. Three of the seven temperament and character variables (harm avoidance, cooperativeness, and self-transcendence) were significantly heritable and were included in subsequent analyses. One endophenotype (high harm avoidance) was able to differentiate the probands from the first-degree relatives and normal controls. A second endophenotype (low self-transcendence) differentiated the controls from the probands and the first-degree relatives. A third endophenotype (cooperativeness) differentiated among all three of the groups, exhibiting the expected order of average group scores. There were no genetic correlations with the schizophrenic phenotype for any dimension of temperament or character. An environmental correlation was suggested for harm avoidance and cooperativeness.

This study replicates previous results that found some personality dimensions to be heritable and familial in families with schizophrenia and offers some promising endophenotypic markers for schizophrenia, although several limitations involving the recruitment of families and phenotyping should be acknowledged. ${ }^{35,36)}$ These endophenotypic markers will serve as important coefficients in solving the mysterious equations that determine schizophrenia. However, the usefulness of most types of positional genetic variations and environmental factors is in doubt as far as their potential to serve as loaded variables in equations describing the causes of the schizophrenic syndrome.

We may speculate that high degrees of harm avoidance plausibly result in negative symptoms because some schizophrenic patients show a tendency towards abulia, alogia, and avolition. With respect to high self-transcendence, this dimension may promote abundant spirituality and creativity; however, at its pathological extreme, excessive self-transcendence may leave patients isolated in an autistic and closed world, leading to psychotic fantasies. Psychotic patients often eventually exhibit low cooperativeness and effectiveness in social settings. They frequently lose their jobs due to such ineffectiveness in social contexts, which results in their overall deterioration. ${ }^{37)}$

Most importantly, future genome-wide linkage and association studies with extensive pedigrees are required. Once such studies have been conducted, it will be possible to perform Family-Based Association Study (FBAT) analyses in families with subtyped schizophrenic probands with respect to personality, working memory, and cognition in addition to the quantitative dimensions described in the present study. Such fine mapping analyses will facilitate the search for candidate genes involved in schizophrenia, a Goliath that currently confronts psychiatrists.

\section{- Acknowledgments}

This study was supported by Pusan National University Grant.

\section{REFERENCES}

1. McGuffin P, Asherson P, Owen M, Farmer A. The strength of the genetic effect. Is there room for an environmental influence in the aetiology of schizophrenia? Br J Psychiatry 1994;164:593-599.

2. Bachmann RF, Schloesser RJ, Gould TD, Manji HK. Mood stabilizers target cellular plasticity and resilience cascades: implications for the development of novel therapeutics. Mol Neurobiol 2005;32:173-202.

3. Bartels SJ, Clark RE, Peacock WJ, Dums AR, Pratt SI. Medicare and medicaid costs for schizophrenia patients by 
age cohort compared with costs for depression, dementia, and medically ill patients. Am J Geriatr Psychiatry 2003; 11:648-657.

4. Bauer M, Unützer J, Pincus HA, Lawson WB; NIMH Affective Disorders Workgroup. Bipolar disorder. Ment Health Serv Res 2002;4:225-229.

5. Shih RA, Belmonte PL, Zandi PP. A review of the evidence from family, twin and adoption studies for a genetic contribution to adult psychiatric disorders. Int Rev Psychiatry 2004; 16:260-283.

6. Sanders AR, Duan J, Levinson DF, Shi J, He D, Hou C, et al. No significant association of 14 candidate genes with schizophrenia in a large European ancestry sample: implications for psychiatric genetics. Am J Psychiatry 2008;165: 497-506.

7. Goate A, Chartier-Harlin MC, Mullan M, Brown J, Crawford F, Fidani L, et al. Segregation of a missense mutation in the amyloid precursor protein gene with familial Alzheimer's disease. Nature 1991;349:704-706.

8. Miki Y, Swensen J, Shattuck-Eidens D, Futreal PA, Harshman $\mathrm{K}$, Tavtigian $\mathrm{S}$, et al. A strong candidate for the breast and ovarian cancer susceptibility gene BRCA1. Science 1994; 266:66-71.

9. MacKinnon DF, Xu J, McMahon FJ, Simpson SG, Stine OC, McInnis MG, et al. Bipolar disorder and panic disorder in families: an analysis of chromosome 18 data. Am J Psychiatry 1998; 155:829-831.

10. McMahon FJ, Simpson SG, McInnis MG, Badner JA, MacKinnon DF, DePaulo JR. Linkage of bipolar disorder to chromosome $18 q$ and the validity of bipolar II disorder. Arch Gen Psychiatry 2001;58:1025-1031.

11. Potash JB, Zandi PP, Willour VL, Lan TH, Huo Y, Avramopoulos D, et al. Suggestive linkage to chromosomal regions $13 q 31$ and $22 q 12$ in families with psychotic bipolar disorder. Am J Psychiatry 2003;160:680-686.

12. Park N, Juo SH, Cheng R, Liu J, Loth JE, Lilliston B, et al. Linkage analysis of psychosis in bipolar pedigrees suggests novel putative loci for bipolar disorder and shared susceptibility with schizophrenia. Mol Psychiatry 2004; 9:1091-1099.

13. Willour VL, Zandi PP, Badner JA, Steele J, Miao K, Lopez $\mathrm{V}$, et al. Attempted suicide in bipolar disorder pedigrees: evidence for linkage to 2p12. Biol Psychiatry 2007;61: 725-727.

14. Raybould R, Green EK, MacGregor S, Gordon-Smith K, Heron J, Hyde S, et al. Bipolar disorder and polymorphisms in the dysbindin gene (DTNBP1). Biol Psychiatry 2005; 57:696-701.

15. Schulze TG, McMahon FJ. Defining the phenotype in human genetic studies: forward genetics and reverse phenotyping. Hum Hered 2004;58:131-138.

16. Freimer N, Sabatti C. The human phenome project. Nat Genet 2003;34:15-21.

17. Singer E. "Phenome" project set to pin down subgroups of autism. Nat Med 2005;11:583.

18. Fresán A, León-Ortiz P, Robles-García R, Azcárraga M, Guizar D, Reyes-Madrigal F, et al. Personality features in ultra-high risk for psychosis: a comparative study with schizophrenia and control subjects using the Temperament and Character Inventory-Revised (TCI-R). J Psychiatr Res 2015;61:168-173.

19. Nitzburg GC, Malhotra AK, DeRosse P. The relationship between temperament and character and subclinical psychotic-like experiences in healthy adults. Eur Psychiatry 2014;29:352-357.
20. Ohi K, Hashimoto R, Yasuda Y, Fukumoto M, Yamamori $\mathrm{H}$, Iwase $\mathrm{M}$, et al. Personality traits and schizophrenia: evidence from a case-control study and meta-analysis. Psychiatry Res 2012;198:7-11.

21. Hori H, Noguchi H, Hashimoto R, Nakabayashi T, Saitoh $\mathrm{O}$, Murray RM, et al. Personality in schizophrenia assessed with the Temperament and Character Inventory (TCI). Psychiatry Res 2008;160:175-183.

22. Teraishi T, Hori H, Sasayama D, Matsuo J, Ogawa S, Ishida $\mathrm{I}$, et al. Personality in remitted major depressive disorder with single and recurrent episodes assessed with the Temperament and Character Inventory. Psychiatry Clin Neurosci 2015;69:3-11.

23. Hasin D, Fenton MC, Skodol A, Krueger R, Keyes K, Geier $\mathrm{T}$, et al. Personality disorders and the 3-year course of alcohol, drug, and nicotine use disorders. Arch Gen Psychiatry 2011;68:1158-1167.

24. Hicks BM, Durbin CE, Blonigen DM, Iacono WG, McGue M. Relationship between personality change and the onset and course of alcohol dependence in young adulthood. Addiction 2012;107:540-548.

25. Hinrichs J, Defife J, Westen D. Personality subtypes in adolescent and adult children of alcoholics: a two-part study. J Nerv Ment Dis 2011;199:487-498.

26. Fassino S, Amianto F, Rocca G, Daga GA. Parental bonding and eating psychopathology in bulimia nervosa: personality traits as possible mediators. Epidemiol Psichiatr Soc 2010;19:214-222.

27. Bora E, Veznedaroglu B. Temperament and character dimensions of the relatives of schizophrenia patients and controls: the relationship between schizotypal features and personality. Eur Psychiatry 2007;22:27-31.

28. Remington G. Alterations of dopamine and serotonin transmission in schizophrenia. Prog Brain Res 2008;172:117-140.

29. Zink M, Correll CU. Glutamatergic agents for schizophrenia: current evidence and perspectives. Expert Rev Clin Pharmacol 2015;8:335-352.

30. Margetić BA, Jakovljević M. Psychobiological model of personality and psychopharmacotherapy outcomes in treatment of depression and schizophrenia. Psychiatr Danub 2013;25:324-328.

31. Cloninger CR. A practical way to diagnosis personality disorder: a proposal. J Pers Disord 2000;14:99-108.

32. Nurnberger JI Jr, Blehar MC, Kaufmann CA, York-Cooler C, Simpson SG, Harkavy-Friedman J, et al. Diagnostic interview for genetic studies. Rationale, unique features, and training. NIMH Genetics Initiative. Arch Gen Psychiatry 1994;51:849-859; discussion 863-864.

33. Maxwell ME. Family Interview for Genetic Studies (FIGS): Manual For FIGS (Clinical Neurogenetics Branch, Intramural Research Program). Bethesda, MD:National Institute of Mental Health;1992.

34. Walss-Bass C, Escamilla MA, Raventos H, Montero AP, Armas R, Dassori A, et al. Evidence of genetic overlap of schizophrenia and bipolar disorder: linkage disequilibrium analysis of chromosome 18 in the Costa Rican population. Am J Med Genet B Neuropsychiatr Genet 2005;139B: 54-60.

35. Sim M, Kim JH, Yim SJ, Cho SJ, Kim SJ. Increase in harm avoidance by genetic loading of schizophrenia. Compr Psychiatry 2012;53:372-378.

36. Smith MJ, Cloninger CR, Harms MP, Csernansky JG. Temperament and character as schizophrenia-related endophenotypes in non-psychotic siblings. Schizophr Res 2008; 104:198-205. 
37. Song YY, Kang JI, Kim SJ, Lee MK, Lee E, An SK. Temperament and character in individuals at ultra-high risk for psychosis and with first-episode schizophrenia: associations with psychopathology, psychosocial functioning, and aspects of psychological health. Compr Psychiatry 2013;54:11611168. 